\title{
Adaptive Control for Linearly and Nonlinearly Parameterized Dynamic Uncertainties in Bilateral Teleoperation Systems
}

\author{
X. Liu and M. Tavakoli
}

\begin{abstract}
Existing work concerning adaptive control of uncertain teleoperation systems can only deal with linearly parameterized (LP) dynamic uncertainties. Typical teleoperation system dynamics, however, posses terms with nonlinearly parameterized (NLP) structures. Stribeck friction is an example of NLP terms in robot dynamics. If not compensated for in the control scheme, uncertainties in the NLP dynamic terms may lead to significant tracking errors. In this paper, for a bilateral teleoperation system, adaptive controllers are designed for the master and slave robots with both LP and NLP dynamic uncertainties. Next, these controllers are incorporated into the 4-channel bilateral teleoperation framework. Then, transparency of the overall teleoperation is studied via a Lyapunov function analysis. A simulation study demonstrates the effectiveness of the proposed adaptive scheme.
\end{abstract}

\section{INTRODUCTION}

$\mathrm{M}$ aster-slave teleoperation systems have been widely applied in many specific areas such as outer space and undersea exploration, minimally invasive telesurgery, nuclear waste site or radioactive material management, and so on. For precise teleoperation, transparency is essential, i.e., the slave must exactly reproduce the master's position trajectory and the master must accurately transmit the slave-environment contact force to the operator so that he/she has the same sensation that the slave does. However, uncertain dynamics is one of the important sources of transparency limitations in teleoperation systems.

In order to address this problem, different adaptive control methods have been sought to mitigate the parametric uncertainties in the dynamics of teleoperation systems. Adaptive control schemes for linear master and slave models have been developed in [1]-[4]. On the other hand, since robot dynamics are generally nonlinear, adaptive control for nonlinear master and slave models is more desirable and challenging compared to those for linear ones. For the nonlinear dynamics case, adaptive control laws were designed for the master and for the slave in [5]-[11].

In terms of the structure of model uncertainties, all of the adaptive teleoperation control work to date have only considered linearly parameterized (LP) dynamic terms (i.e., terms involving model parameters that appear linearly in the

This research was supported by the Natural Sciences and Engineering Research Council (NSERC) of Canada under grant RGPIN-372042 and by the China Scholarship Council (CSC) under grant [2009]3012.

$\mathrm{X}$. Liu is with the School of Automation Engineering, University of Electronic Science and Technology of China, Chengdu, Sichuan, 611731 China. She is now a visiting PhD student in the Department of Electrical and Computer Engineering, University of Alberta, Edmonton, Alberta, T6G 2V4 Canada. (e-mail: xia8@ualberta.ca).

M. Tavakoli is with the Department of Electrical and Computer Engineering, University of Alberta, Edmonton, Alberta, T6G 2V4 Canada. (e-mail: tavakoli@ece.ualberta.ca). dynamics) and have not considered nonlinearly parameterized (NLP) dynamic terms. Such NLP dynamic uncertainties in the master and/or slave robots of a teleoperation system degrade position and force tracking and, therefore, degrade transparency if they are not compensated for in the control scheme. Friction, which is ubiquitous in the joints of the master and slave robots, is an example of NLP terms [12]. Indeed, at the $i$ th joint of a robot, the friction force can be

modeled as

$$
\mu_{c i} \operatorname{sgn}\left(\dot{q}_{i}\right)+\mu_{v i} \dot{q}_{i}+\left(\mu_{s i}-\mu_{c i}\right) \operatorname{sgn}\left(\dot{q}_{i}\right) \exp \left(-\frac{\dot{q}_{i}^{2}}{v_{s i}^{2}}\right)
$$

where $q_{i}$ is the joint angle position, $\mu_{c i}, \mu_{v i}, \mu_{s i}$ are the coefficients of Coulomb friction, viscous friction, and static friction, respectively, and $v_{s i}$ is the Stribeck velocity. Note that $\mu_{c i}, \mu_{v i}, \mu_{s i}$ and $v_{s i}$ are potentially uncertain parameters, and $v_{s i}$ appears nonlinearly in the dynamics.

To compensation for uncertain NLP terms, Feemster et al. [13] designed an adaptive controller to compensate for uncertainties in the parameters appearing nonlinearly in the friction model. However, the result in [13] can only be applied to setpoint regulation. For trajectory tracking, Hung et al. [14] developed an adaptive controller to compensate for NLP uncertainties in robot manipulators. The approach in [14] has only been applied to motion control of a single robot in free motion. So far, there has been no attempt at simultaneous motion and force control in a master-slave teleoperation system with NLP uncertainties. Motivated by this fact, the contribution of this paper is in adaptive control of a bilateral teleoperation system encompassing both LP and NLP dynamic uncertainties. The proposed adaptive controllers for the master and for the slave are then incorporated into the 4-channel bilateral framework to achieve transparent teleoperation.

The organization of this paper is as follows. In Section II, the nonlinear model of a teleoperation system is developed. In Section III, adaptive controllers which can deal with LP and NLP dynamic uncertainties are designed for the master and for the slave, and incorporated into the 4-channel bilateral teleoperation control framework. Then, transparency of the entire closed-loop system is studied by a Lyapunov function analysis. In Section IV, computer simulations are presented comparing the performance of the proposed adaptive controller with that of a conventional adaptive controller. The paper is concluded in Section V. 


\section{MODEL OF A TELEOPERATION SYSTEM WITH NLP DYNAMIC TERMS}

When interacting with a human operator and an environment, the task-space nonlinear dynamic models of $n$-DOF master and the slave robots can be written as

$$
\begin{gathered}
\mathbf{M}_{x m}\left(\mathbf{q}_{m}\right) \ddot{\mathbf{x}}_{m}+\mathbf{C}_{x m}\left(\mathbf{q}_{m}, \dot{\mathbf{q}}_{m}\right) \dot{\mathbf{x}}_{m}+\mathbf{G}_{x m}\left(\mathbf{q}_{m}\right) \\
-\mathbf{N}_{x m}\left(\mathbf{q}_{m}, \dot{\mathbf{q}}_{m}, \boldsymbol{\eta}_{m}\right)=\mathbf{f}_{m}+\mathbf{f}_{h} \\
\mathbf{M}_{x s}\left(\mathbf{q}_{s}\right) \ddot{\mathbf{x}}_{s}+\mathbf{C}_{x s}\left(\mathbf{q}_{s}, \dot{\mathbf{q}}_{s}\right) \dot{\mathbf{x}}_{s}+\mathbf{G}_{x s}\left(\mathbf{q}_{s}\right) \\
-\mathbf{N}_{x s}\left(\mathbf{q}_{s}, \dot{\mathbf{q}}_{s}, \mathbf{\eta}_{s}\right)=\mathbf{f}_{s}-\mathbf{f}_{e}
\end{gathered}
$$

where $\mathbf{q}_{m}$ and $\mathbf{q}_{s} \in \mathfrak{R}^{n \times 1}$ are joint angle positions, $\mathbf{x}_{m}$ and $\mathbf{x}_{s} \in \mathfrak{R}^{6 \times 1}$ are end-effector Cartesian positions/orientations, $\mathbf{M}_{x m}\left(\mathbf{q}_{m}\right) \quad$ and $\quad \mathbf{M}_{x s}\left(\mathbf{q}_{s}\right) \in \mathfrak{R}^{6 \times 6}$ are symmetric positive-definite inertia matrices, $\mathbf{C}_{x m}\left(\mathbf{q}_{m}, \dot{\mathbf{q}}_{m}\right)$ and $\mathbf{C}_{x s}\left(\mathbf{q}_{s}, \dot{\mathbf{q}}_{s}\right) \in \mathfrak{R}^{6 \times 6}$ correspond to Coriolis and centrifugal terms, $\mathbf{G}_{x m}\left(\mathbf{q}_{m}\right)$ and $\mathbf{G}_{x s}\left(\mathbf{q}_{s}\right) \in \mathfrak{R}^{6 \times 1}$ represent gravity terms, $\mathbf{f}_{m}$ and $\mathbf{f}_{s} \in \mathfrak{R}^{6 \times 1}$ are force/torque control inputs, and $\mathbf{f}_{h} \in \mathfrak{R}^{6 \times 1}$ is the force/torque that the operator applies to the master, $\mathbf{f}_{e} \in \mathfrak{R}^{6 \times 1}$ is the force/torque that the environment applies to the slave. Also, $\mathbf{N}_{x m}\left(\mathbf{q}_{m}, \dot{\mathbf{q}}_{m}, \mathbf{\eta}_{m}\right)$ and $\mathbf{N}_{x s}\left(\mathbf{q}_{s}, \dot{\mathbf{q}}_{s}, \boldsymbol{\eta}_{s}\right) \in \mathfrak{R}^{6 \times 1}$ represent terms whose parameters $\boldsymbol{\eta}_{m}$ and $\boldsymbol{\eta}_{s}$ are uncertain and appear nonlinearly in the model, and are assumed to have the multiplicative form

$$
N_{x i}\left(\mathbf{q}, \dot{\mathbf{q}}, \boldsymbol{\eta}_{i}\right)=R_{i}\left(\mathbf{q}, \dot{\mathbf{q}}, \boldsymbol{\eta}_{i}\right) T_{i}\left(\mathbf{q}, \dot{\mathbf{q}}, \boldsymbol{\eta}_{i}\right)
$$

Here and after, the subscripts $m$ and $s$ (for master and slave) are omitted in the properties for brevity. For the $i^{\text {th }}$ joint of each robot, $R_{i}\left(\mathbf{q}, \dot{\mathbf{q}}, \boldsymbol{\eta}_{i}\right)$ and $T_{i}\left(\mathbf{q}, \dot{\mathbf{q}}, \boldsymbol{\eta}_{i}\right)$ are nonlinear functions assumed to be Lipschitz in $\boldsymbol{\eta}_{i}=\left[\eta_{i 1, \ldots,}, \eta_{i p_{i}}\right]^{T} \in \mathfrak{R}^{p_{i} \times 1}[14]:$

$$
\begin{gathered}
\left\|R_{i}\left(\mathbf{q}, \dot{\mathbf{q}}, \boldsymbol{\eta}_{i}\right)-R_{i}\left(\mathbf{q}, \dot{\mathbf{q}}, \tilde{\boldsymbol{\eta}}_{i}\right)\right\| \\
\leq \sum_{j=1}^{p_{i}} A_{i j}(\mathbf{q}, \dot{\mathbf{q}})\left|\boldsymbol{\eta}_{i j}-\tilde{\boldsymbol{\eta}}_{i j}\right|, \forall \boldsymbol{\eta}_{i}, \tilde{\boldsymbol{\eta}}_{i} \in \boldsymbol{\eta} \\
\leq T_{i}\left(\mathbf{q}, \dot{\mathbf{q}}, \boldsymbol{\eta}_{i}\right)-T_{i}\left(\mathbf{q}, \dot{\mathbf{q}}, \tilde{\boldsymbol{\eta}}_{i}\right) \| \\
\leq \sum_{j=1}^{p_{i}} Z_{i j}(\mathbf{q}, \dot{\mathbf{q}})\left|\boldsymbol{\eta}_{i j}-\tilde{\boldsymbol{\eta}}_{i j}\right|, \forall \boldsymbol{\eta}_{i}, \tilde{\boldsymbol{\eta}}_{i} \in \boldsymbol{\eta}
\end{gathered}
$$

where $A_{i j}(\mathbf{q}, \dot{\mathbf{q}}), Z_{i j}(\mathbf{q}, \dot{\mathbf{q}}) \geq 0$ are continuous functions.

Property 1 [15]. The first three terms in the left sides of dynamics (1) and (2) are of LP structure:

$$
\mathbf{M}_{x}(\mathbf{q}) \ddot{\mathbf{x}}+\mathbf{C}_{x}(\mathbf{q}, \dot{\mathbf{q}}) \dot{\mathbf{x}}+\mathbf{G}_{x}(\mathbf{q})=\mathbf{Y}(\mathbf{q}, \dot{\mathbf{q}}, \dot{\mathbf{x}}, \ddot{\mathbf{x}}) \boldsymbol{\alpha}
$$

where $\boldsymbol{\alpha} \in \mathfrak{R}^{n \times r}$ is the dynamic LP parameter vector, and $\mathbf{Y} \in \mathfrak{R}^{n \times r}$ is a regressor matrix.

Property 2 [15]. The matrix $\dot{\mathbf{M}}_{x}(\mathbf{q})-2 \mathbf{C}_{x}(\mathbf{q}, \dot{\mathbf{q}})$ is skew-symmetric:

$$
\zeta^{T}\left(\dot{\mathbf{M}}_{x}(\mathbf{q})-2 \mathbf{C}_{x}(\mathbf{q}, \dot{\mathbf{q}})\right) \xi=0, \forall \xi \in \mathfrak{R}^{n \times 1}
$$

Property 3 [14]. The NLP terms in (1) and (2) satisfy

$$
\mathbf{s}^{T} \mathbf{N}_{x}(\mathbf{q}, \dot{\mathbf{q}}, \boldsymbol{\eta}) \leq \mathbf{s}^{T} \mathbf{N}_{x}(\mathbf{q}, \dot{\mathbf{q}}, \mathbf{0})+\mathbf{s}^{T} \mathbf{Q}(\mathbf{s}, \mathbf{q}, \dot{\mathbf{q}}) \boldsymbol{\theta}
$$

where $\mathbf{s}=\Delta \dot{\mathbf{x}}+\boldsymbol{\lambda} \Delta \mathbf{x}, \Delta \mathbf{x}$ is the position error between the actual position and the desired position, $\Delta \dot{\mathbf{x}}$ is the velocity error, $\lambda$ is a diagonal positive definite matrix, and

$$
\begin{aligned}
& \boldsymbol{\theta}:=\left[\begin{array}{llll}
\boldsymbol{\theta}_{1}^{T} & \boldsymbol{\theta}_{2}^{T} & \ldots & \boldsymbol{\theta}_{n}^{T}
\end{array}\right]^{T} \in \mathfrak{R}^{2 n \times 1} \\
& \boldsymbol{\theta}_{i}:=\left[\left(\sum_{j=1}^{p_{i}} \eta_{i j}\right)^{2} \quad \sum_{j=1}^{p_{i}} \eta_{i j}\right]^{T} \in \mathfrak{R}^{2 \times 1} \\
& \mathbf{Q}(\mathbf{s}, \mathbf{q}, \dot{\mathbf{q}}):=\operatorname{diag}\left[\operatorname{sgn}\left(s_{1}\right) \mathbf{w}_{1}(\mathbf{q}, \dot{\mathbf{q}}), \ldots,\right. \\
& \left.\operatorname{sgn}\left(s_{n}\right) \mathbf{w}_{n}(\mathbf{q}, \dot{\mathbf{q}})\right] \in \mathfrak{R}^{n \times 2 n} \\
& \mathbf{w}_{i}:=\left[A_{i}(\mathbf{q}, \dot{\mathbf{q}}) Z_{i}(\mathbf{q}, \dot{\mathbf{q}}) \quad\left\|T_{i}(\mathbf{q}, \dot{\mathbf{q}}, \mathbf{0})\right\| A_{i}(\mathbf{q}, \dot{\mathbf{q}})\right. \\
& \left.+\left\|R_{i}(\mathbf{q}, \dot{\mathbf{q}}, \mathbf{0})\right\| Z_{i}(\mathbf{q}, \dot{\mathbf{q}})\right] \in \mathfrak{R}^{1 \times 2} \\
& A_{i}(\mathbf{q}, \dot{\mathbf{q}}):=\max _{j=1,2, \ldots, p_{i}} A_{i j}(\mathbf{q}, \dot{\mathbf{q}}) \\
& Z_{i}(\mathbf{q}, \dot{\mathbf{q}}):=\max _{j=1,2, \ldots, p_{i}} Z_{i j}(\mathbf{q}, \dot{\mathbf{q}})
\end{aligned}
$$

\section{ADAPTIVE BILATERAL TELEOPERATION CONTROL}

In an ideally transparent teleoperation system, through appropriate control signals, the master and the slave positions and forces will match regardless of the operator and environment dynamics, i.e., $\mathbf{x}_{m}=\mathbf{x}_{s}, \mathbf{f}_{h}=\mathbf{f}_{e}$. To achieve this ideal response, the 4-channel teleoperation control architectures is the most successful one [16]-[18]. Thus, the basic idea of our proposed teleoperation control scheme is based on incorporating two adaptive position controllers for the master and the slave into the 4-channel teleoperation architecture.

Our goal is to design a control scheme for a teleoperation system with both LP and NLP dynamic uncertainties (i.e., uncertainties both in $\boldsymbol{\alpha}_{m}$ and $\boldsymbol{\alpha}_{s}$, and in $\boldsymbol{\eta}_{m}$ and $\boldsymbol{\eta}_{s}$ ) such that good transparency is achieved. To do this, the fixed position controllers for the master and for the slave in the original 4-channel teleoperation architecture are replaced by two adaptive position controllers, respectively. Other controllers in the original 4-channel teleoperation architecture are still utilized as force feedforward and feedback controllers in the proposed approach.

\section{A. Design of Control Laws and Adaptation Laws}

Similar to the definition of $\mathbf{s}$ in Property 3, define two vectors $\mathbf{s}_{m}, \mathbf{s}_{s} \in \mathfrak{R}^{n \times 1}$ in the task space for the master and for the slave, respectively:

$$
\begin{aligned}
& \mathbf{s}_{m}=\Delta \dot{\mathbf{x}}_{m}+\lambda \Delta \mathbf{x}_{m}=\dot{\mathbf{x}}_{m}-\dot{\mathbf{x}}_{m r} \\
& \mathbf{s}_{s}=\Delta \dot{\mathbf{x}}_{s}+\lambda \Delta \mathbf{x}_{s}=\dot{\mathbf{x}}_{s}-\dot{\mathbf{x}}_{s r}
\end{aligned}
$$

where

$$
\begin{aligned}
\Delta \mathbf{x}_{m} & =\mathbf{x}_{m}-\mathbf{x}_{s}, \Delta \mathbf{x}_{s}=\mathbf{x}_{s}-\mathbf{x}_{m} \\
\dot{\mathbf{x}}_{m r} & =\dot{\mathbf{x}}_{s}-\lambda \Delta \mathbf{x}_{m}, \dot{\mathbf{x}}_{s r}=\dot{\mathbf{x}}_{m}-\lambda \Delta \mathbf{x}_{s}
\end{aligned}
$$


From (4)-(5), we get

$$
\begin{array}{ll}
\dot{\mathbf{x}}_{m}=\mathbf{s}_{m}+\dot{\mathbf{x}}_{m r}, & \dot{\mathbf{x}}_{s}=\mathbf{s}_{s}+\dot{\mathbf{x}}_{s r} \\
\ddot{\mathbf{x}}_{m}=\dot{\mathbf{s}}_{m}+\ddot{\mathbf{x}}_{m r}, & \ddot{\mathbf{x}}_{s}=\dot{\mathbf{s}}_{s}+\ddot{\mathbf{x}}_{s r}
\end{array}
$$

Substituting (6)-(7) into (1)-(2), the open-loop dynamics of the master and the slave become

$$
\begin{gathered}
\mathbf{M}_{x m}\left(\mathbf{q}_{m}\right) \dot{\mathbf{s}}_{m}+\mathbf{C}_{x m}\left(\mathbf{q}_{m}, \dot{\mathbf{q}}_{m}\right) \mathbf{s}_{m}=\mathbf{f}_{m}+\mathbf{f}_{h} \\
-\mathbf{Y}_{m r}\left(\mathbf{q}_{m}, \dot{\mathbf{q}}_{m}, \dot{\mathbf{x}}_{m r}, \ddot{\mathbf{x}}_{m r}\right) \boldsymbol{\alpha}_{m}+\mathbf{N}_{x m}\left(\mathbf{q}_{m}, \dot{\mathbf{q}}_{m}, \mathbf{\eta}_{m}\right) \\
\quad \mathbf{M}_{x s}\left(\mathbf{q}_{s}\right) \dot{\mathbf{s}}_{s}+\mathbf{C}_{x s}\left(\mathbf{q}_{s}, \dot{\mathbf{q}}_{s}\right) \mathbf{s}_{s}=\mathbf{f}_{s}-\mathbf{f}_{e} \\
-\mathbf{Y}_{s r}\left(\mathbf{q}_{s}, \dot{\mathbf{q}}_{s}, \dot{\mathbf{x}}_{s r}, \ddot{\mathbf{x}}_{s r}\right) \boldsymbol{\alpha}_{s}+\mathbf{N}_{x s}\left(\mathbf{q}_{s}, \dot{\mathbf{q}}_{s}, \boldsymbol{\eta}_{s}\right)
\end{gathered}
$$

where

$$
\begin{gathered}
\mathbf{Y}_{m r}\left(q_{m}, \dot{\mathbf{q}}_{m}, \dot{\mathbf{x}}_{m r}, \ddot{\mathbf{x}}_{m r}\right) \boldsymbol{\alpha}_{m} \\
=\mathbf{M}_{x m}\left(\mathbf{q}_{m}\right) \ddot{\mathbf{x}}_{m r}+\mathbf{C}_{x m}\left(\mathbf{q}_{m}, \dot{\mathbf{q}}_{m}\right) \dot{\mathbf{x}}_{m r}+\mathbf{G}_{x m}\left(\mathbf{q}_{m}\right) \\
\mathbf{Y}_{s r}\left(\mathbf{q}_{s}, \dot{\mathbf{q}}_{s}, \dot{\mathbf{x}}_{s r}, \ddot{\mathbf{x}}_{s r}\right) \boldsymbol{\alpha}_{s} \\
=\mathbf{M}_{x s}\left(q_{s}\right) \ddot{\mathbf{x}}_{s r}+\mathbf{C}_{x s}\left(\mathbf{q}_{s}, \dot{\mathbf{q}}_{s}\right) \dot{\mathbf{x}}_{s r}+\mathbf{G}_{x s}\left(\mathbf{q}_{s}\right)
\end{gathered}
$$

Now, the control laws and the adaptation laws for the master and the slave are proposed as the following:

- Control laws:

$$
\begin{aligned}
\mathbf{f}_{m}=- & \mathbf{K}_{m} \mathbf{s}_{m}+\mathbf{Y}_{m r} \hat{\boldsymbol{\alpha}}_{m}-\mathbf{N}_{x m}\left(\mathbf{q}_{m}, \dot{\mathbf{q}}_{m}, \mathbf{0}\right) \\
& -\mathbf{Q}_{m} \hat{\boldsymbol{\theta}}_{m}+\mathbf{C}_{2}\left(\mathbf{f}_{h}-\mathbf{f}_{e}\right)-\mathbf{f}_{h} \\
\mathbf{f}_{s}=- & \mathbf{K}_{s} \mathbf{s}_{s}+\mathbf{Y}_{s r} \hat{\boldsymbol{\alpha}}_{s}-\mathbf{N}_{x s}\left(\mathbf{q}_{s}, \dot{\mathbf{q}}_{s}, \mathbf{0}\right) \\
& -\mathbf{Q}_{s} \hat{\boldsymbol{\theta}}_{s}+\mathbf{C}_{3}\left(\mathbf{f}_{h}-\mathbf{f}_{e}\right)+\mathbf{f}_{e}
\end{aligned}
$$

where $\mathbf{K}_{m}, \mathbf{K}_{s}, \mathbf{C}_{2}$ and $\mathbf{C}_{3}$ are diagonal positive-definite matrices (or positive constants), and $\hat{\boldsymbol{\alpha}}_{m}, \hat{\boldsymbol{\alpha}}_{s}, \hat{\boldsymbol{\theta}}_{m}, \hat{\boldsymbol{\theta}}_{s}$ are estimates of $\boldsymbol{\alpha}_{m}, \boldsymbol{\alpha}_{s}, \boldsymbol{\theta}_{m}, \boldsymbol{\theta}_{s}$, respectively. Note that $\mathbf{Q}_{m}$, $\mathbf{Q}_{s}, \boldsymbol{\theta}_{m}$ and $\boldsymbol{\theta}_{s}$ have been defined in Property 3.

Each of the control laws (12)-(13) includes six terms. The first term is a feedback law for velocity and position tracking between the master and the slave, the second term compensates for the LP dynamic uncertainties, and the third and the fourth terms compensate for the NLP dynamic uncertainties. These first four terms together perform adaptive position control. The fifth term implements force tracking between the master and the slave (i.e., force feedback to the operator), and the sixth term cancels the human/master or the slave/environment interaction force in the corresponding dynamics. The estimated LP parameters $\hat{\boldsymbol{\alpha}}_{m}$ and $\hat{\boldsymbol{\alpha}}_{s}$, and the estimated NLP parameters $\hat{\boldsymbol{\theta}}_{m}$ and $\hat{\boldsymbol{\theta}}_{s}$ are updated by the following adaptation laws:

- Adaptation laws for the LP uncertain parameters in dynamics:

$$
\dot{\hat{\boldsymbol{\alpha}}}_{m}=-\boldsymbol{\Gamma}_{a m} \mathbf{Y}_{m r}^{T} \mathbf{s}_{m}, \dot{\hat{\boldsymbol{\alpha}}}_{s}=-\boldsymbol{\Gamma}_{a s} \mathbf{Y}_{s r}^{T} \mathbf{s}_{s}
$$

where $\boldsymbol{\Gamma}_{\alpha m}$ and $\boldsymbol{\Gamma}_{\alpha s}$ are constant positive-definite matrices. - Adaptation laws for the NLP uncertain parameters in dynamics:

$$
\dot{\hat{\boldsymbol{\theta}}}_{m}=\boldsymbol{\Gamma}_{\theta m} \mathbf{Q}_{m}^{T} \mathbf{s}_{m}, \dot{\hat{\boldsymbol{\theta}}}_{s}=\boldsymbol{\Gamma}_{\theta s} \mathbf{Q}_{s}^{T} \mathbf{s}_{s}
$$

where $\boldsymbol{\Gamma}_{\theta m}$ and $\boldsymbol{\Gamma}_{\theta s}$ are constant positive-definite matrices.
We are now in a position to find the closed-loop system dynamics. Substituting the control laws (12)-(13) into the open-loop dynamics (8)-(9), the closed-loops dynamics for the master and the slave are obtained as

$$
\begin{gathered}
\quad \mathbf{M}_{x m}\left(\mathbf{q}_{m}\right) \dot{\mathbf{s}}_{m}+\mathbf{C}_{x m}\left(\mathbf{q}_{m}, \dot{\mathbf{q}}_{m}\right) \mathbf{s}_{m} \\
=-\mathbf{K}_{m} \mathbf{s}_{m}+\mathbf{Y}_{m r} \Delta \boldsymbol{\alpha}_{m}+\mathbf{N}_{x m}\left(\mathbf{q}_{m}, \dot{\mathbf{q}}_{m}, \boldsymbol{\eta}_{m}\right) \\
-\mathbf{N}_{x m}\left(\mathbf{q}_{m}, \dot{\mathbf{q}}_{m}, \mathbf{0}\right)-\mathbf{Q}_{m} \hat{\boldsymbol{\theta}}_{m}+\mathbf{C}_{2}\left(\mathbf{f}_{h}-\mathbf{f}_{e}\right) \\
\quad \mathbf{M}_{x s}\left(\mathbf{q}_{s}\right) \dot{\mathbf{s}}_{s}+\mathbf{C}_{x s}\left(\mathbf{q}_{s}, \dot{\mathbf{q}}_{s}\right) \mathbf{s}_{s} \\
=-\mathbf{K}_{s} \mathbf{s}_{s}+\mathbf{Y}_{s r} \Delta \boldsymbol{\alpha}_{s}+\mathbf{N}_{x s}\left(\mathbf{q}_{s}, \dot{\mathbf{q}}_{s}, \boldsymbol{\eta}_{s}\right) \\
-\mathbf{N}_{x s}\left(\mathbf{q}_{s}, \dot{\mathbf{q}}_{s}, \mathbf{0}\right)-\mathbf{Q}_{s} \hat{\boldsymbol{\theta}}_{s}+\mathbf{C}_{3}\left(\mathbf{f}_{h}-\mathbf{f}_{e}\right)
\end{gathered}
$$

where $\Delta \boldsymbol{\alpha}_{m}=\hat{\boldsymbol{\alpha}}_{m}-\boldsymbol{\alpha}_{m}$ and $\Delta \boldsymbol{\alpha}_{s}=\hat{\boldsymbol{\alpha}}_{s}-\boldsymbol{\alpha}_{s}$. Multiplying both sides of (17) by $\mathbf{C}_{2} \mathbf{C}_{3}^{-1}$, subtracting the result from (16), and using $\mathbf{s}_{s}=-\mathbf{s}_{m}$ gives us a unified closed-loop equation for the entire master-slave system as

$$
\begin{aligned}
& \left(\mathbf{M}_{x m}\left(\mathbf{q}_{m}\right)+\mathbf{C}_{2} \mathbf{C}_{3}{ }^{-1} \mathbf{M}_{x s}\left(\mathbf{q}_{s}\right)\right) \dot{\mathbf{s}}_{m} \\
& +\left(\mathbf{C}_{x m}\left(\mathbf{q}_{m}, \dot{\mathbf{q}}_{m}\right)+\mathbf{C}_{2} \mathbf{C}_{3}{ }^{-1} \mathbf{C}_{x s}\left(\mathbf{q}_{s}\right)\right) \mathbf{s}_{m} \\
= & -\left(\mathbf{K}_{m}+\mathbf{C}_{2} \mathbf{C}_{3}{ }^{-1} \mathbf{K}_{s}\right) \mathbf{s}_{m}+\mathbf{Y}_{m r} \Delta \boldsymbol{\alpha}_{m} \\
+ & +\mathbf{N}_{x m}\left(\mathbf{q}_{m}, \dot{\mathbf{q}}_{m}, \mathbf{\eta}_{m}\right)-\mathbf{N}_{x m}\left(\mathbf{q}_{m}, \dot{\mathbf{q}}_{m}, \mathbf{0}\right)-\mathbf{Q}_{m} \hat{\boldsymbol{\theta}}_{m} \\
& -\mathbf{C}_{2} \mathbf{C}_{3}^{-1} \mathbf{Y}_{s r} \Delta \boldsymbol{\alpha}_{s}-\mathbf{C}_{2} \mathbf{C}_{3}{ }^{-1} \mathbf{N}_{x s}\left(\mathbf{q}_{s}, \dot{\mathbf{q}}_{s}, \boldsymbol{\eta}_{s}\right) \\
& +\mathbf{C}_{2} \mathbf{C}_{3}{ }^{-1} \mathbf{N}_{x s}\left(\mathbf{q}_{s}, \dot{\mathbf{q}}_{s}, \mathbf{0}\right)+\mathbf{C}_{2} \mathbf{C}_{3}{ }^{-1} \mathbf{Q}_{s} \hat{\boldsymbol{\theta}}_{s}
\end{aligned}
$$

Remark 1: In the controllers (12)-(13), switching activities may exist because of the discontinuous function $\operatorname{sgn}()$ in $\mathbf{Q}_{m}$ and $\mathbf{Q}_{s}$, which may be undesirable. This can be alleviated by replacing $\operatorname{sgn}()$ with a smooth saturation function, e.g.,

$$
s_{i}=\left\{\begin{array}{lll}
1 & \text { if } & s_{i}>\varepsilon \\
-1 & \text { if } & s_{i}<\varepsilon \\
\frac{s_{i}}{\varepsilon} & \text { if } & -\varepsilon<s_{i}<\varepsilon
\end{array}\right.
$$

where $\varepsilon$ is a small positive constant.

\section{B. Transparency of the Closed-loop Teleoperation System}

In this section, we will use a unified Lyapunov function to study the transparency of the entire teleoperation system.

Theorem 1: Consider that the nonlinear teleoperation system (1)-(2) has both LP and NLP dynamic uncertainties and is controlled by the adaptive controller (12)-(13) using the LP dynamic adaptation laws (14) and the NLP dynamic adaptation laws (15). Assume the matrices $\mathbf{C}_{2}$ and $\mathbf{C}_{3}$ are invertible. Then, $\mathbf{s}_{m}, \Delta \boldsymbol{\alpha}_{m}, \Delta \boldsymbol{\theta}_{m}, \Delta \boldsymbol{\alpha}_{s}$ and $\Delta \boldsymbol{\theta}_{s}$ are bounded. Moreover, the position tracking error $\Delta \mathbf{x}_{m}=\mathbf{x}_{m}-\mathbf{x}_{s}$ converges to zero as $t \rightarrow \infty$. Also, the force tracking error $\Delta \mathbf{f}=\mathbf{f}_{m}-\mathbf{f}_{s}$ is bounded.

Proof: Consider the Lyapunov function candidate 


$$
\begin{aligned}
V= & \frac{1}{2} \mathbf{s}_{m}^{T}\left(\mathbf{M}_{x m}\left(\mathbf{q}_{m}\right)+\mathbf{C}_{2} \mathbf{C}_{3}{ }^{-1} \mathbf{M}_{x s}\left(\mathbf{q}_{s}\right)\right) \mathbf{s}_{m} \\
& +\frac{1}{2} \Delta \boldsymbol{\alpha}_{m}^{T} \boldsymbol{\Gamma}_{\alpha m}^{-1} \Delta \boldsymbol{\alpha}_{m}+\frac{1}{2} \Delta \boldsymbol{\theta}_{m}^{T} \boldsymbol{\Gamma}_{\theta m}^{-1} \Delta \boldsymbol{\theta}_{m} \\
& +\frac{1}{2} \mathbf{C}_{2} \mathbf{C}_{3}^{-1} \Delta \boldsymbol{\alpha}_{s}^{T} \boldsymbol{\Gamma}_{\alpha s}^{-1} \Delta \boldsymbol{\alpha}_{s} \\
& +\frac{1}{2} \mathbf{C}_{2} \mathbf{C}_{3}^{-1} \Delta \boldsymbol{\theta}_{s}^{T} \boldsymbol{\Gamma}_{\theta s}^{-1} \Delta \boldsymbol{\theta}_{s}
\end{aligned}
$$

where $\Delta \boldsymbol{\theta}_{m}=\hat{\boldsymbol{\theta}}_{m}-\boldsymbol{\theta}_{m}$ and $\Delta \boldsymbol{\theta}_{s}=\hat{\boldsymbol{\theta}}_{s}-\boldsymbol{\theta}_{s}$. Differentiating $V$ along the trajectory of the unified closed-loop system (18), using Property 2 , and $\mathbf{s}_{s}=-\mathbf{s}_{m}$ gives

$$
\begin{aligned}
\dot{V}= & -\mathbf{s}_{m}^{T}\left(\mathbf{K}_{m}+\mathbf{C}_{2} \mathbf{C}_{3}{ }^{-1} \mathbf{K}_{s}\right) \mathbf{s}_{m}+\mathbf{s}_{m}^{T} \mathbf{Y}_{m r} \Delta \boldsymbol{\alpha}_{m} \\
& +\mathbf{s}_{m}^{T}\left(\mathbf{N}_{x m}\left(\mathbf{q}_{m}, \dot{\mathbf{q}}_{m}, \boldsymbol{\eta}_{m}\right)-\mathbf{N}_{x m}\left(\mathbf{q}_{m}, \dot{\mathbf{q}}_{m}, \mathbf{0}\right)\right. \\
& \left.-\mathbf{Q}_{m} \hat{\boldsymbol{\theta}}_{m}\right)+\mathbf{s}_{s}^{T} \mathbf{C}_{2} \mathbf{C}_{3}{ }^{-1} \mathbf{Y}_{s r} \Delta \boldsymbol{\alpha}_{s}+\mathbf{s}_{s}^{T} \mathbf{C}_{2} \mathbf{C}_{3}{ }^{-1} \\
& \left(\mathbf{N}_{x s}\left(\mathbf{q}_{s}, \dot{\mathbf{q}}_{s}, \boldsymbol{\eta}_{s}\right)-\mathbf{N}_{x s}\left(\mathbf{q}_{s}, \dot{\mathbf{q}}_{s}, \mathbf{0}\right)-\mathbf{Q}_{s} \hat{\boldsymbol{\theta}}_{s}\right) \\
& +\Delta \boldsymbol{\alpha}_{m}^{T} \boldsymbol{\Gamma}_{\alpha m}^{-1} \dot{\hat{\boldsymbol{\alpha}}}_{m}+\Delta \boldsymbol{\theta}_{m}^{T} \boldsymbol{\Gamma}_{\theta m}^{-1} \dot{\hat{\boldsymbol{\theta}}}_{m} \\
& +\mathbf{C}_{2} \mathbf{C}_{3}{ }^{-1} \Delta \boldsymbol{\alpha}_{s}^{T} \boldsymbol{\Gamma}_{\alpha s}^{-1} \dot{\hat{\boldsymbol{\alpha}}}_{s}+\mathbf{C}_{2} \mathbf{C}_{3}{ }^{-1} \Delta \boldsymbol{\theta}_{s}^{T} \boldsymbol{\Gamma}_{\theta s}^{-1} \dot{\hat{\boldsymbol{\theta}}}_{s}
\end{aligned}
$$

Using Property $3,(20)$ becomes

$$
\begin{aligned}
\dot{V} \leq & -\mathbf{s}_{m}^{T}\left(\mathbf{K}_{m}+\mathbf{C}_{2} \mathbf{C}_{3}{ }^{-1} \mathbf{K}_{s}\right) \mathbf{s}_{m}+\mathbf{s}_{m}^{T} \mathbf{Y}_{m r} \Delta \boldsymbol{\alpha}_{m} \\
& -\mathbf{s}_{m}^{T} \mathbf{Q}_{m} \Delta \boldsymbol{\theta}_{m}+\mathbf{s}_{s}^{T} \mathbf{C}_{2} \mathbf{C}_{3}{ }^{-1} \mathbf{Y}_{s r} \Delta \boldsymbol{\alpha}_{s} \\
& -\mathbf{s}_{s}^{T} \mathbf{C}_{2} \mathbf{C}_{3}{ }^{-1} \mathbf{Q}_{s} \Delta \boldsymbol{\theta}_{s}+\Delta \boldsymbol{\alpha}_{m}^{T} \boldsymbol{\Gamma}_{\alpha m}^{-1} \dot{\hat{\boldsymbol{\alpha}}}_{m} \\
& +\Delta \boldsymbol{\theta}_{m}^{T} \boldsymbol{\Gamma}_{\theta m}^{-1} \dot{\hat{\boldsymbol{\theta}}}_{m}+\mathbf{C}_{2} \mathbf{C}_{3}{ }^{-1} \Delta \boldsymbol{\alpha}_{s}^{T} \boldsymbol{\Gamma}_{\alpha s}^{-1} \dot{\boldsymbol{\alpha}}_{s} \\
& +\mathbf{C}_{2} \mathbf{C}_{3}{ }^{-1} \Delta \boldsymbol{\theta}_{s}^{T} \boldsymbol{\Gamma}_{\theta s}^{-1} \dot{\hat{\boldsymbol{\theta}}}_{s}
\end{aligned}
$$

Substituting the adaptation laws (14)-(15) into (21), we obtain

$$
\dot{V} \leq-\mathbf{s}_{m}^{T}\left(\mathbf{K}_{m}+\mathbf{C}_{2} \mathbf{C}_{3}{ }^{-1} \mathbf{K}_{s}\right) \mathbf{s}_{m}
$$

From (19) and (22), we find that $V$ is positive-definite and $\dot{V}$ is negative semi-definite. Therefore, $V$ is bounded. Hence, $\mathbf{s}_{m}, \Delta \boldsymbol{\alpha}_{m}, \Delta \boldsymbol{\theta}_{m}, \Delta \boldsymbol{\alpha}_{s}$ and $\Delta \boldsymbol{\theta}_{s}$ are bounded.

In terms of position tracking, since $\mathbf{s}_{m}=\Delta \dot{\mathbf{x}}_{m}+\lambda \Delta \mathbf{x}_{m}$ is a BIBO stable system with a pole at $-\lambda$, the boundedness of $\mathbf{s}_{m}$ results in the boundedness of $\Delta \mathbf{x}_{m}$. With $\mathbf{s}_{m}$ and $\Delta \mathbf{x}_{m}$ bounded, it is imperative that $\Delta \dot{\mathbf{x}}_{m}$ is also bounded. Moreover, from (22), we have

$$
\begin{aligned}
& e i g_{\min }\left(\mathbf{K}_{m}\right) \mathbf{s}_{m}^{T} \mathbf{s}_{m}+e i g_{\min }\left(\mathbf{C}_{2} \mathbf{C}_{3}{ }^{-1} \mathbf{K}_{s}\right) \mathbf{s}_{m}^{T} \mathbf{s}_{m} \\
\leq & \mathbf{s}_{m}^{T}\left(\mathbf{K}_{m}+\mathbf{C}_{2} \mathbf{C}_{3}{ }^{-1} \mathbf{K}_{s}\right) \mathbf{s}_{m} \leq-\dot{V}
\end{aligned}
$$

where $e i g_{\min }\left(\mathbf{K}_{m}\right)$ and $e i g_{\min }\left(\mathbf{C}_{2} \mathbf{C}_{3}{ }^{-1} \mathbf{K}_{s}\right)$ denote the minimum eigenvalues of matrices $\mathbf{K}_{m}$ and $\mathbf{C}_{2} \mathbf{C}_{3}{ }^{-1} \mathbf{K}_{s}$, respectively.

Integrating both sides of (23), we get

$$
\begin{aligned}
& e i g_{\min }\left(\mathbf{K}_{m}\right) \int_{0}^{t}\left\|\mathbf{s}_{m}\right\|^{2} d t+e i g_{\min }\left(\mathbf{C}_{2} \mathbf{C}_{3}^{-1} \mathbf{K}_{s}\right) \int_{0}^{t}\left\|\mathbf{s}_{s}\right\|^{2} d t \\
\leq & -\int_{0}^{t} \dot{V} d t=V(0)-V(t) \leq V(0)<\infty
\end{aligned}
$$

Hence, $\quad \mathbf{s}_{m}=\Delta \dot{\mathbf{x}}_{m}+\lambda \Delta \mathbf{x}_{m} \in L_{2}$ and followed by $\Delta \mathbf{x}_{m} \in L_{2}$. According to Barbalat's Lemma [15], we can get $\Delta \mathbf{x}_{m} \rightarrow 0$ as $t \rightarrow \infty$.

In terms of force tracking, we already have that $\mathbf{s}_{m}, \Delta \boldsymbol{\alpha}_{m}, \Delta \boldsymbol{\theta}_{m}, \Delta \boldsymbol{\alpha}_{s}, \Delta \boldsymbol{\theta}_{s}$ are bounded and, according to the unified closed-loop dynamics (18) and Property 3, we have

$$
\begin{aligned}
& \left(\mathbf{M}_{x m}\left(\mathbf{q}_{m}\right)+\mathbf{C}_{2} \mathbf{C}_{3}^{-1} \mathbf{M}_{x s}\left(\mathbf{q}_{s}\right)\right) \dot{\mathbf{s}}_{m} \\
\leq & -\left(\mathbf{C}_{x m}\left(\mathbf{q}_{m}, \dot{\mathbf{q}}_{m}\right)+\mathbf{C}_{2} \mathbf{C}_{3}{ }^{-1} \mathbf{C}_{x s}\left(\mathbf{q}_{s}\right)\right) \mathbf{s}_{m} \\
& -\left(\mathbf{K}_{m}+\mathbf{C}_{2} \mathbf{C}_{3}{ }^{-1} \mathbf{K}_{s}\right) \mathbf{s}_{m}+\mathbf{Y}_{m r} \Delta \boldsymbol{\alpha}_{m}-\mathbf{Q}_{m} \Delta \boldsymbol{\theta}_{m} \\
& -\mathbf{C}_{2} \mathbf{C}_{3}{ }^{-1} \mathbf{Y}_{s r} \Delta \boldsymbol{\alpha}_{s}+\mathbf{C}_{2} \mathbf{C}_{3}{ }^{-1} \mathbf{Q}_{s} \Delta \boldsymbol{\theta}_{s}
\end{aligned}
$$

Thus, $\dot{\mathbf{s}}_{m}$ is bounded. Furthermore, according to (16) and Property 3, we have

$$
\begin{aligned}
\mathbf{C}_{2}\left(\mathbf{f}_{h}-\mathbf{f}_{e}\right) \leq & \mathbf{M}_{x m}\left(\mathbf{q}_{m}\right) \dot{\mathbf{s}}_{m}+\mathbf{C}_{x m}\left(\mathbf{q}_{m}, \dot{\mathbf{q}}_{m}\right) \mathbf{s}_{m} \\
& +\mathbf{K}_{m} \mathbf{s}_{m}-\mathbf{Y}_{m r} \Delta \boldsymbol{\alpha}_{m}+\mathbf{Q}_{m} \Delta \boldsymbol{\theta}_{m}
\end{aligned}
$$

Therefore, $\Delta \mathbf{f}=\mathbf{f}_{h}-\mathbf{f}_{e}$ is bounded as $t \rightarrow \infty$. This concludes the proof.

\section{SimUlation STUDIES}

In this section, simulations are done to demonstrate the validity of the proposed adaptive control scheme. We take two identical rotational-prismatic (RP) manipulators as the master and the slave robots, shown in fig. 1. The inertial, Coriolis and centrifugal, and gravity terms and Jacobian matrix of a RP manipulator are [19]:

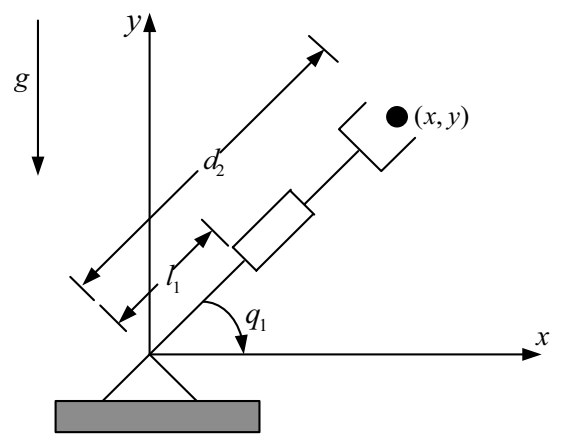

Fig. 1. Rotational-prismatic robot

$$
\begin{gathered}
\mathbf{M}(\mathbf{q})=\left[\begin{array}{cc}
m_{1} l_{1}^{2}+m_{2} d_{2}^{2} & 0 \\
0 & m_{2}
\end{array}\right], \\
\mathbf{C}(\mathbf{q}, \dot{\mathbf{q}})=\left[\begin{array}{cc}
0 & 2 m_{2} d_{2} \dot{q}_{1} \\
-m_{2} d_{2} \dot{q}_{1} & 0
\end{array}\right], \\
\mathbf{G}(\mathbf{q})=\left[\begin{array}{c}
\left(m_{1} l_{1}+m_{2} d_{2}\right) g \cos \left(q_{1}\right) \\
m_{2} g \sin \left(q_{1}\right)
\end{array}\right], \\
\mathbf{J}=\left[\begin{array}{cc}
-d_{2} \sin q_{1} & \cos q_{1} \\
d_{2} \cos q_{1} & \sin q_{1}
\end{array}\right],
\end{gathered}
$$


where $l_{1}$ is the length of the first link, $m_{1}$ and $m_{2}$ are the point masses of the links, $g$ is the gravity constant, $q_{1}$ is the joint angle corresponding to the rotary joint, $d_{2}$ is a variable distance corresponding to the position of the prismatic joint, and $\mathbf{q}=\left[q_{1}, d_{2}\right]^{T}$. After transforming the dynamics from the joint space to the Cartesian space, the LP parameter vector $\boldsymbol{\alpha}$ can be found as $\boldsymbol{\alpha}=\left[m_{1} l_{1}^{2}, m_{2}, m_{1} l_{1} g, m_{2} g\right]^{T}$. Then, according to Property $1, \mathbf{Y}$ can be found.

For the NLP term, we consider the Stribeck friction effect, i.e., $\left(\mu_{s i}-\mu_{c i}\right) \operatorname{sgn}\left(\dot{q}_{i}\right) \exp \left(-\frac{\dot{q}_{i}^{2}}{v_{s i}^{2}}\right)$. The NLP parameter vectors $\boldsymbol{\theta}_{m}, \boldsymbol{\theta}_{s}$ can be found as $\boldsymbol{\theta}_{m}=\boldsymbol{\theta}_{s}=\left[\theta_{1}, \theta_{2}, \theta_{3}, \theta_{4}\right]^{T}$, where

$$
\begin{gathered}
\theta_{1}=\theta_{3}=\left(\mu_{s 1}-\mu_{c 1}+\mu_{s 2}-\mu_{c 2}+\frac{1}{v_{s 1}^{2}}+\frac{1}{v_{s 2}^{2}}\right)^{2} \\
\theta_{2}=\theta_{4}=\mu_{s 1}-\mu_{c 1}+\mu_{s 2}-\mu_{c 2}+\frac{1}{v_{s 1}^{2}}+\frac{1}{v_{s 2}^{2}}
\end{gathered}
$$

Then according to Property $3, \mathbf{Q}_{m}$ and $\mathbf{Q}_{s}$ can be found.

The robot parameters [19], the friction parameters [14], and the controller parameters are shown in Table 1.

\begin{tabular}{cccc}
\multicolumn{4}{c}{ TABle 1: PARAMETERS OF THE ROBOTS, FRICTION TERM } \\
AND CONTROLLER \\
\hline \hline$m_{1}$ & $m_{2}$ & $l_{1}$ & $g$ \\
$4.6 \mathrm{~kg}$ & $2.3 \mathrm{~kg}$ & $0.5 m$ & $9.8 \mathrm{kgm} / \mathrm{s}^{2}$ \\
$\mu_{s i}$ & $\mu_{c i}$ & $v_{s i}$ & $\boldsymbol{\lambda}$ \\
3.5 & 0.49 & 0.189 & $0.1 \mathbf{I}$ \\
$\mathbf{K}_{m}, \mathbf{K}_{s}$ & $\boldsymbol{\Gamma}_{\alpha m}, \boldsymbol{\Gamma}_{\alpha s}$ & $\boldsymbol{\Gamma}_{\theta m}, \boldsymbol{\Gamma}_{\theta s}$ & $\mathbf{C}_{2}, \mathbf{C}_{3}$ \\
$10 \mathbf{I}$ & $0.05 \mathbf{I}$ & $0.05 \mathbf{I}$ & $300 \mathbf{I}$ \\
\hline \hline
\end{tabular}

In the simulations, the human operator and environment are modeled as

$$
\begin{aligned}
& \mathbf{f}_{h}=\mathbf{f}_{h}^{*}-\left(m_{h} \ddot{\mathbf{x}}_{m}+b_{h} \dot{\mathbf{x}}_{m}+k_{h} \mathbf{x}_{m}\right), \\
& \mathbf{f}_{e}=m_{e} \ddot{\mathbf{x}}_{s}+b_{e} \dot{\mathbf{x}}_{s}+k_{e} \mathbf{x}_{s},
\end{aligned}
$$

where

$$
\begin{aligned}
& m_{h}=3.25 \mathrm{~kg}, b_{h}=20 \mathrm{Nsm}^{-1}, k_{h}=300 \mathrm{Nm}^{-1}, \\
& m_{e}=1 \mathrm{~kg}, b_{e}=40 \mathrm{Nsm}^{-1}, k_{e}=1500 \mathrm{Nm}^{-1}
\end{aligned}
$$

are coefficients corresponding to the mass, damping, and stiffness of the operator's hand and the environment, respectively. Besides, $\mathbf{f}_{h}^{*}=[\sin t, 0]^{T}$ denotes the exogenous forces of the operator.

According to Table 1, the true parameter vectors are

$$
\begin{aligned}
& \boldsymbol{\alpha}_{m}=\boldsymbol{\alpha}_{s}=[1.15,2.3,22.54,22.54]^{T} \\
& \boldsymbol{\theta}_{m}=\boldsymbol{\theta}_{s}=[3845.2,62,3845.2,62]^{T} .
\end{aligned}
$$

The initial estimates of $\boldsymbol{\alpha}_{m}, \boldsymbol{\alpha}_{s}, \boldsymbol{\theta}_{m}$ and $\boldsymbol{\theta}_{s}$ are randomly set as

$$
\begin{gathered}
\hat{\boldsymbol{\alpha}}_{m}(0)=\hat{\boldsymbol{\alpha}}_{s}(0)=[0.92,1.84,18.032,18.032]^{T} \\
\hat{\boldsymbol{\theta}}_{m}(0)=\hat{\boldsymbol{\theta}}_{s}(0)=[1922.6,31,1922.6,31]^{T} .
\end{gathered}
$$

Also, the robots initial positions are set as

$$
\mathbf{x}_{m}(0)=\mathbf{x}_{s}(0)=[0.4,0.6928]^{T} .
$$

The proposed adaptive control scheme is compared with a well-known conventional adaptive control scheme [6] that can only deal with LP dynamic uncertainties. The simulation results in $\mathrm{x}$-direction are shown in Fig. 2 and Fig. 3. From Fig. 2 , we can see that for the proposed adaptive control, the position trajectories of the master and the slave are very close to one another, and the force tracking error is bounded and quite small, as the method is meant to compensate for uncertain $\boldsymbol{\alpha}_{m}, \boldsymbol{\alpha}_{s}, \boldsymbol{\theta}_{m}$ and $\boldsymbol{\theta}_{s}$. Comparatively, from Fig. 3, we can see that for the conventional adaptive control, the position tracking is not perfect and the force tracking error is bigger, as the method cannot compensate for the NLP uncertainties in $\boldsymbol{\theta}_{m}$ and $\boldsymbol{\theta}_{s}$. Note that since the exogenous force in the y-direction is zero $\left(\mathbf{f}_{h}^{*}=[\sin t, 0]^{T}\right)$, the positions and forces are not shown in that direction.

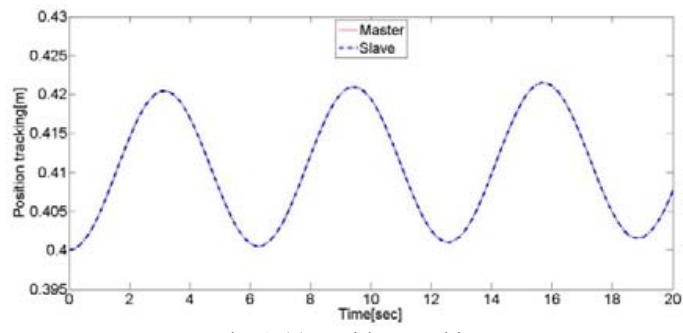

Fig. 2 (a) Position tracking

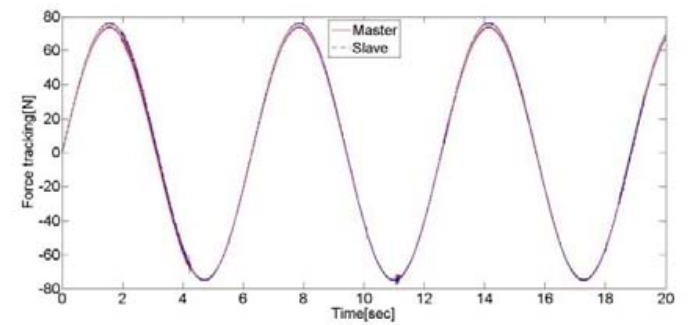

Fig. 2 (b) Force tracking

Fig. 2 Proposed adaptive control scheme, which deals with both LP and NLP dynamic uncertainties.

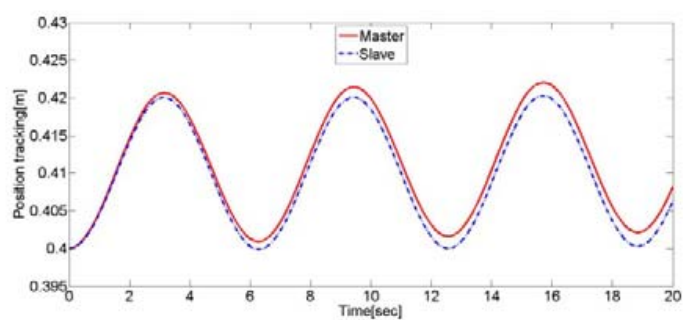

Fig. 3 (a) Position tracking 


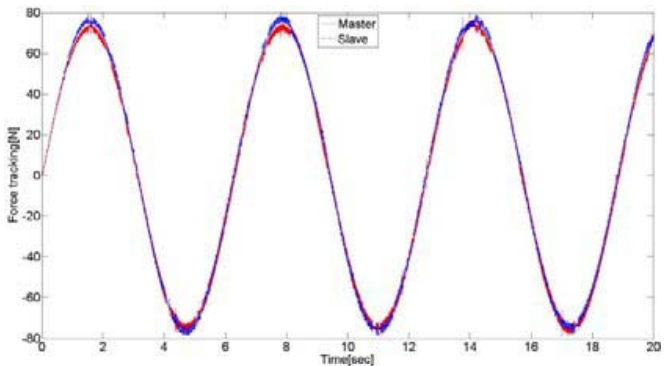

Fig. 3 (b) Force tracking

Fig. 3 Conventional adaptive control scheme, which merely deals with LP dynamic uncertainties.

Remark 2: It is worth noting that the estimated parameters $\hat{\boldsymbol{\alpha}}_{m}, \hat{\boldsymbol{\alpha}}_{s}, \hat{\boldsymbol{\theta}}_{m}, \hat{\boldsymbol{\theta}}_{s}$ do not need to converge to their true values $\boldsymbol{\alpha}_{m}, \boldsymbol{\alpha}_{s}, \boldsymbol{\theta}_{m}, \boldsymbol{\theta}_{s}$. A key point in adaptive control is that the tracking errors of the system may converge to zero regardless of whether the parameter errors converge to zero or not.

\section{CONCLUSIONS}

In this paper, for a bilateral teleoperation system, adaptive controllers are designed for the master and slave robots with both LP and NLP dynamic uncertainties. The controllers are incorporated into the 4-channel bilateral teleoperation framework. Transparency of the overall teleoperation is studied via a Lyapunov function analysis and demonstrated by simulation studies. Compared with the conventional adaptive control scheme which cannot deal with NLP dynamic uncertainties, the proposed control scheme has better position and force tracking performance despite there are deviations both in the LP and NLP uncertain terms.

With respect to position tracking, we have achieved that the position tracking error $\Delta \mathbf{x}_{m}=\mathbf{x}_{m}-\mathbf{x}_{s}$ converges to zero as $t \rightarrow \infty$, while for force tracking, we have shown the force tracking error $\Delta \mathbf{f}=\mathbf{f}_{h}-\mathbf{f}_{e}$ is bounded. Improving the present control algorithm to ensure that the force tracking error can converge to zero remains as future work. In addition, time delay is assumed to be negligible in this paper, therefore accounting for communication time delay especially variable delay will also be addressed in our future work.

\section{REFERENCES}

[1] K.H. Zaad, and S.E., Salcudean, "Adaptive Impedance Reflecting Teleoperation," in Proceedings of the IEEE International Conference on Robotics and Automation, Minnesota, USA, April, pp. 1369-1374, 1996.

[2] Hyoung-Ki Lee and M. J. Chung, "Adaptive controller of a master-slave system for transparent teleoperation," Journal of Robotic systems, vol. 15, no. 8, pp. 465-475, 1998.

[3] M. Shi, G. Tao and H. Liu, "Adaptive control of teleoperation systems," Journal of X-Ray Science and Technology, vol. 10, no. 1-2, pp. 37-57, 2002.

[4] K.B. Fite, M. Goldfarb, and A. Rubio, "Loop Shaping for Transparency and Stability Robustness in Time-delayed Bilateral Telemanipulation," Journal of Dynamic Systems, Measurement and Control, Transactions of the ASME, Vol. 126, no. 3, pp. 650-656, 2004.

[5] Wen-Hong Zhu and S. E. Salcudean, "Stability guaranteed teleoperation: an adaptive motion/force control approach," IEEE transactions on automatic control, vol. 45, no. 11, pp. 1951-1969,
2000

[6] J. H. Ryu and D. S. Kwon, "A novel adaptive bilateral control scheme using similar closed-loop dynamic characteristics of master/slave manipulators," Journal of Robotic Systems, vol. 18, no. 9, pp. 533-543, 2001.

[7] N. V. Q. Hung, T. Narikiyo and H. D. Tuan, "Nonlinear adaptive control of master-slave system in teleoperation," Control Engineering Practice, vol. 11, no. 1, pp. 1-10, 2003.

[8] I.G. Polushin, A. Tayebi, H. J. Marquez, "Adaptive schemes for stable teleoperation with communication delay based on IOS small gain theorem," Proceedings of the American Control Conference, Vol. 6, pp. 4143-4148, Portland, OR, USA, 2005.

[9] S. Sirouspour and P. Setoodeh, "Adaptive nonlinear teleoperation control in multi-master/multi-slave environments," in Proceeding of the IEEE Conference on Control Applications, Toronto, Canada, August, pp. 1263-1268, 2005.

[10] N. Chopra, M. W. Spong and R. Lozano, "Synchronization of bilateral teleoperators with time delay," Automatica, vol. 44, no. 8, pp. 2142-2148, 2008

[11] E. Nuño, R. Ortega and L. Basañez, "An adaptive controller for nonlinear teleoperators," Automatica, vol. 46, no. 1, pp. 155-159, 2010.

[12] B. Armstrong-Helouvry, P. Dupont, and C. Canudas de Wit, "A survey of models, analysis tools and compensation methods for control of machines with friction," Automatica, vol. 30, no. 6, pp. 1083-1138, 1994.

[13] M. Feemster, P. Vedagarbha, D. M. Dawson and D. Haste, "Adaptive control techniques for friction compensation," Mechatronics, vol. 9, no. 2, pp.125-145, 1999.

[14] N. V. Q. Hung, H. D. Tuan, T. Narikiyo and P. Apkarian, "Adaptive control for nonlinearly parameterized uncertainties in robot," IEEE transactions on control systems technology, vol.16, no.3, pp.458-468, 2008.

[15] J. J. E. Slotine and W. Li, Applied nonlinear control, Prentice-Hall, Englewood Cliffs, NJ, 1991.

[16] D. A. Lawrence, "Stability and transparency in bilateral teleoperation," IEEE Transactions on Robotics and Automation, vol. 9, no. 5, pp. 624-637, 1993

[17] Y. Yokokohji and T. Yoshikawa, "Bilateral control of master-slave manipulator for ideal kinesthetic coupling-formulation and experiment," IEEE Transactions on Robotics and Automation, vol. 10, no. 5, pp. 605-619, 1994.

[18] M. Tavakoli, A. Aziminejad, R.V. Patel and M. Moallem, "High-fidelity bilateral teleoperation systems and the effect of multimodal haptics," IEEE Transactions on Systems, Man, and Cybernetics - Part B, vol. 37, no.6, pp. 1512-1528, 2007.

[19] J. Craig, Introduction to robotics: mechanics and control ( $3^{\text {rd }} \mathrm{Ed}$.), Pearson Prentice Hall, USA, 2005. 